\title{
CONSTRUÇÃO DE CURVAS DE TEMPERATURAS PARA A VISCOSIDADE E DENSIDADE DAS BLENDAS FORMADAS COM DIESEL MINERAL E BIODIESEL DE COCO, DENDE E OURICURI
}

\author{
R. GABRIEL ${ }^{1}$, A. K.S. LIMA ${ }^{1}$, G. E. S. SANTOS ${ }^{1}$, J. I. SOLETTI ${ }^{1}$ e S. H. V. de CARVALHO ${ }^{1}$, L. \\ MEILI $^{1}$ \\ ${ }^{1}$ Universidade Federal de Alagoas, Centro de Tecnologia \\ E-mail para contato: jisoletti @gmail.com
}

\begin{abstract}
RESUMO - A utilização do biodiesel como combustível vem ocorrendo de maneira gradual incentivado pelo Programa Nacional de Produção e Uso de Biodiesel (Lei $\mathrm{n}^{\mathbf{o}}$ 11.097/05). Neste sentido, faz-se necessário conhecer algumas propriedades da mistura do diesel com biodieseis obtidos a partir de diferentes oleaginosas. Neste trabalho, o diesel foi misturado a diferentes biodieseis, produzidos a partir de três óleos vegetais (coco, dendê e ouricuri), em diferentes proporções: B0, B20, B40, B60, B80 e B100. Propriedades fundamentais das misturas, tais como a densidade e viscosidade, foram determinadas, com base nas normas ASTM D664 e ASTM D445, respectivamente, conforme determinação dos parâmetros citados na Resolução da Agência Nacional do Petróleo, Gás Natural e Biocombustíveis (ANP). As propriedades foram determinadas a diferentes temperaturas: $10,15,20,25,30,40,60$ e $80^{\circ} \mathrm{C}$. Foi observado que a densidade e a viscosidade da mistura aumentam com o aumento do percentual do biodiesel nas misturas. Com relação a influência da temperatura,essas propriedades diminuem com o aumento da temperatura, para todas as misturas de biodieseis estudadas.
\end{abstract}

\section{INTRODUÇÃO}

$\mathrm{Na}$ expectativa de diminuir o impacto ambiental causado pela emissão de poluentes gasosos dos combustíveis fósseis, pesquisas têm sido estimuladas para viabilização de fontes de energia renováveis, biodegradáveis, não tóxicas ao meio ambiente e economicamente viáveis. $\mathrm{O}$ biodiesel é a alternativa mais promissora para a substituição total ou parcial do diesel a base de petróleo, devido aos seus benefícios ambientais, Al-Hamamre et al. (2014).

As vantagens do biodiesel tem incentivado a concepção de novas abordagens para a sua produção e de processamento para se obter um combustível de alta qualidade. Em cumprimento à legislação, atualmente vem sendo utilizado misturas de diesel/biodiesel com o objetivo de diminuir as emissões de dióxido de carbono. A porcentagem de biodiesel presente no diesel é adequadamente designada como "misturas de biodiesel", atualmente no Brasil o diesel já é comercializado a mistura B5, Souza et al. (2013), Hoekman et al. (2012), Caires et al. (2012). A mistura desses compostos provocam diferenças nas propriedades físico-químicas e, dessa forma, os parâmetros de qualidade do 

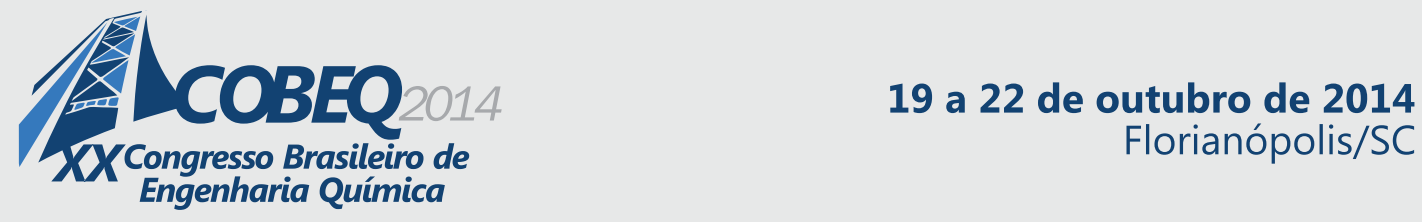

biodiesel são importantes para os usuários finais, pois podem afetar o desempenho do motor e as emissões de poluentes.

Nesta perspectiva, o estudo das propriedades termodinâmicas de biodiesel torna-se crucial uma vez que fornecem informações estratégicas que permitem estabelecer padrões de qualidade que evitem prejudicar a qualidade das emissões da queima, desempenho e a integridade do motor, assim como a segurança no transporte e manuseio do biodiesel , de modo que suas propriedades estão em conformidade com as normas regulamentares Souza et al. (2013), Fernandes et al. (2011). No Brasil, a ANP é responsável pela definição de padrões para a certificação da qualidade do biodiesel.

A Viscosidade cinemática e densidade são uns dos parâmetros exigidos pelas normas de biodiesel e combustível diesel, por serem propriedades-chave de combustível para motores à diesel. São capazes de afetar o início da injeção, a pressão de injeção, e a característica de pulverização de combustível, de modo que eles influenciam as emissões de desempenho do motor de combustão e gases de escape. Altas viscosidades e densidades também causam mais problemas em tempo frio, pois elas aumentam com a diminuição da temperatura. A densidade é uma propriedade do combustível fundamental, pois muitas características de desempenho, tais como o número de cetano e valor de aquecimento, estão relacionados com a ela.

Existe, atualmente, uma série de pesquisas e testes voltados à utilização de blendas de biodiesel/diesel no Brasil e no mundo, Freitas et al. (2014), Fernandes et al. (2011), Caires et al. (2012).

Diante disto, neste trabalho foram preparadas misturas de biodiesel adicionado ao diesel de petróleo. Avaliou-se a viscosidade e a densidade, pelas normas ASTM D445 e ASTM D664, respectivamente, das misturas: B0, B20, B40, B60, B80 e B100 para o biodiesel produzido a partir do óleo de ouricuri, coco, e dendê caracterizados a partir da determinação dos parâmetros físicoquímicos citados na Resolução da Agência Nacional do Petróleo, Gás Natural e Biocombustíveis (ANP), visando avaliar se as misturas estão em conformidade com esta norma.

\section{METODOLOGIA}

O biodiesel utilizado foi produzido no Laboratório de Sistemas de Separação e Otimização de Processos (LASSOP) da Universidade Federal de Alagoas, a partir da reação de transesterificação do óleo de coco, ouricuri e dendê, em uma unidade piloto, composta por um reator encamisado, um agitador mecânico e por um banho termostático. Após a produção e purificação do biodiesel foram realizadas as misturas (blendas) diesel/biodiesel, para as concentrações pré-estabelecidas: B0, B20, B40, B60, B80 e B100, e, posteriormente realizados as análises físico-químicas da viscosidade e densidade:

\subsection{Analise da Viscosidade para Diferentes Blends de Biodiesel}

A viscosidade foi determinada de acordo com a NORMA ASTM D445, utilizando um viscosímetro de Ostwald da marca SCHOTT. Em um capilar com constante de $150 \mathrm{cSt} / \mathrm{s}$ foram 


\section{9 a 22 de outubro de 2014 \\ Florianópolis/SC}

adicionados cerca de $8 \mathrm{~mL}$ de amostra dos blends de biodiesel e determinado o seu tempo de escoamento a para as temperaturas de: 20, 25, 30, 40, 60 e $80{ }^{\circ} \mathrm{C}$. Todos os experimento foram realizados em triplicada.

\subsection{Analise da Densidade para Diferentes Blendas de Biodiesel}

A densidade foi determinada de acordo com a norma ASTM D664, utilizando um densímetro digital (modelo DMA 35N, ANTAN PAAR). A amostra das blendas de biodiesel foi submetida as temperatura de $10,15,20,30$ e $40^{\circ} \mathrm{C}$ e feita à leitura diretamente no densímetro. $\mathrm{O}$ experimento foi realizado em triplicada.

\section{RESULTADOS}

Os resultados obtidos por meio dos ensaios experimentais, para cada mistura biodiesel/diesel das diferentes palmáceas estudadas (coco, ouricuri e dendê), serão subdivididos em duas seções, que descrevem o comportamento da viscosidade e densidades das misturas frente à variação de temperatura do meio. Os parâmetros físico-químicos para o biodiesel puro (B100) e suas misturas com diesel de petróleo (B0, B20, B40, B60 e B80) foram estudadas as viscosidades e densidades com emprego das normas ASTM D445 e ASTM D664, respectivamente.

\subsection{Análises das Viscosidades}

A Viscosidade Cinemática é uma propriedade fluidodinâmica que expressa a resistência oferecida pela substância ao escoamento sob gravidade. Devido à viscosidade indicar a resistência do fluido ao escoamento, o controle dessa propriedade para o biodiesel é importante para garantir funcionamento adequado dos sistemas de injeção e bombas de combustível. A viscosidade também influencia a etapa de combustão, cuja eficiência dependerá da potência máxima desenvolvida pelo motor. Os dados obtidos são apresentados nas Tabelas 1, 2 e 3:

Pela ASTM D445 a viscosidade cinemática para o biodiesel deve estar entre 1,9 e $6,0 \mathrm{~mm}^{2} . \mathrm{s}^{-1} \mathrm{a}$ $40^{\circ} \mathrm{C}$ e para as blendas deve estar entre $1,9 \mathrm{e} 4,1 \mathrm{~mm}^{2} . \mathrm{s}^{-1}$ a $40^{\circ} \mathrm{C}$. A viscosidade dos três biodieseis produzidos, forneceram os valores de $2,5482 \mathrm{~mm}^{2} \mathrm{~s}^{-} ; 4,2744 \mathrm{~mm}^{2} \mathrm{~s}^{-}$e 2,9592 $\mathrm{mm}^{2} \mathrm{~s}^{-}$para os biodiesel de coco, dendê e ouricuri, respectivamente. Portanto, as viscosidades dos três biodieseis estão de acordo com as normas. Observamos a partir da Figura 1,2 e 3, que a viscosidade apresenta um comportamento crescente, indicando que a viscosidade aumenta em razão do aumento de biodiesel na composição da mistura. Este é um comportamento esperado uma vez que um líquido mais viscoso tem sua concentração aumentada em uma dada mistura. Observou-se também que as viscosidades das misturas são afetadas diretamente pela temperatura, o viscosidade diminui a medida que a temperatura aumenta. De um modo geral, a viscosidade diminui com o aumento da temperatura e aumenta com a fração de biodiesel na mistura biodiesel/diesel. 
Tabela 1 - Viscosidade $\left(\mathrm{mm}^{2} / \mathrm{s}\right)$ obtida para os blends biodiesel/diesel de Ouricuri.

\begin{tabular}{ccccccc} 
& B0 & B20 & B40 & B60 & B80 & B100 \\
\hline $\mathbf{2 5}^{\circ} \mathbf{C}$ & 4,508 & 4,7173 & 5,1359 & 5,3613 & 5,474 & 5,5706 \\
$\mathbf{3 0}^{\circ} \mathbf{C}$ & 3,5742 & 4,0411 & 4,2665 & 4,3148 & 4,3953 & 4,4275 \\
$\mathbf{4 0}^{\circ} \mathbf{C}$ & 2,7692 & 3,0429 & 3,38905 & 3,34075 & 3,4776 & 3,6064 \\
$\mathbf{6 0}^{\circ} \mathbf{C}$ & 1,9803 & 2,2379 & 2,34255 & 2,29425 & 2,45203 & 2,4794 \\
$\mathbf{8 0}^{\circ} \mathbf{C}$ & 1,5295 & 1,5617 & 1,69855 & 1,73075 & 1,77905 & 1,85955 \\
\hline
\end{tabular}

Tabela 2 - Viscosidade $\left(\mathrm{mm}^{2} / \mathrm{s}\right)$ obtida para os blends biodiesel/diesel de Coco.

\begin{tabular}{ccccccc} 
& \multicolumn{1}{c}{ B0 } & B20 & B40 & B60 & B80 & B100 \\
\hline $\mathbf{2 0}^{\circ} \mathbf{C}$ & 4,508 & 5,04413 & 5,152 & 5,1681 & 5,24055 & 5,313 \\
$\mathbf{2 5}^{\circ} \mathbf{C}$ & 3,8801 & 4,2665 & 4,4275 & 4,4436 & 4,5563 & 4,61265 \\
$\mathbf{3 0}^{\circ} \mathbf{C}$ & 3,5742 & 3,8962 & 4,0733 & 4,0894 & 4,1055 & 4,1377 \\
$\mathbf{4 0}^{\circ} \mathbf{C}$ & 2,7692 & 2,9141 & 3,0107 & 3,1234 & 3,2039 & 3,3166 \\
$\mathbf{6 0}^{\circ} \mathbf{C}$ & 1,9803 & 2,1896 & 2,2057 & 2,2379 & 2,3184 & 2,3506 \\
$\mathbf{8 0}^{\circ} \mathbf{C}$ & 1,5295 & 1,6422 & 1,72753 & 1,73075 & 1,75973 & 1,771 \\
\hline
\end{tabular}

Tabela 3 - Viscosidade ( $\left.\mathrm{mm}^{2} / \mathrm{s}\right)$ obtida para os blends biodiesel/diesel de Dênde.

\begin{tabular}{ccccccc} 
& B0 & B20 & B40 & B60 & B80 & B100 \\
\hline $\mathbf{2 0}^{\circ} \mathbf{C}$ & 4,508 & 6,075 & 6,762 & 7,614 & 7,857 & 8,05 \\
$\mathbf{2 5}^{\circ} \mathbf{C}$ & 3,8801 & 5,1198 & 6,2694 & 6,3917 & 7,128 & 7,1484 \\
$\mathbf{3 0}^{\circ} \mathbf{C}$ & 3,5742 & 4,4275 & 5,2325 & 5,6028 & 5,9409 & 6,3434 \\
$\mathbf{4 0}^{\circ} \mathbf{C}$ & 2,7692 & 3,1717 & 3,6225 & 4,0411 & 4,5563 & 5,1037 \\
$\mathbf{6 0}^{\circ} \mathbf{C}$ & 1,9803 & 2,3184 & 2,6726 & 2,898 & 3,1878 & 3,4454 \\
$\mathbf{8 0}^{\circ} \mathbf{C}$ & 1,5295 & 1,7549 & 2,0447 & 2,2218 & 2,3345 & 2,4955 \\
\hline
\end{tabular}

As Figuras 1, 2 e 3 apresentam os resultados obtidos a partir do estudo das viscosidades das misturas de diesel/ biodiesel das oleaginosas estudadas conforme as concentrações pré-estabelecidas: B20, B40, B60, B80, B100 e diesel. 


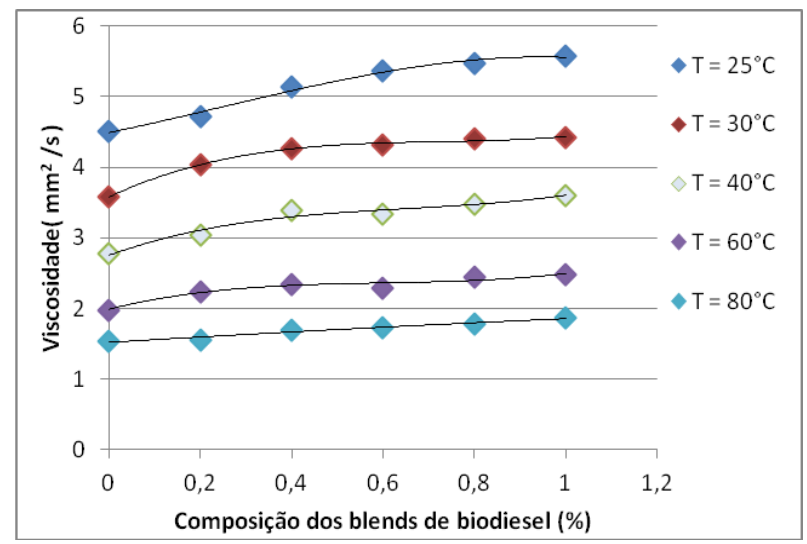

Figura 1 - Curva de viscosidade dos blends de diesel/ biodiesel de ouricuri.

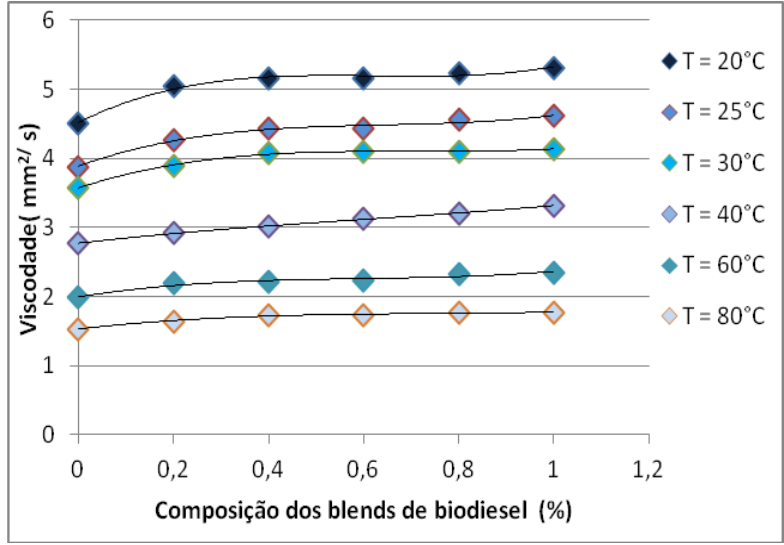

Figura 2 - Curva de viscosidade dos blends de diesel/ biodiesel de coco.

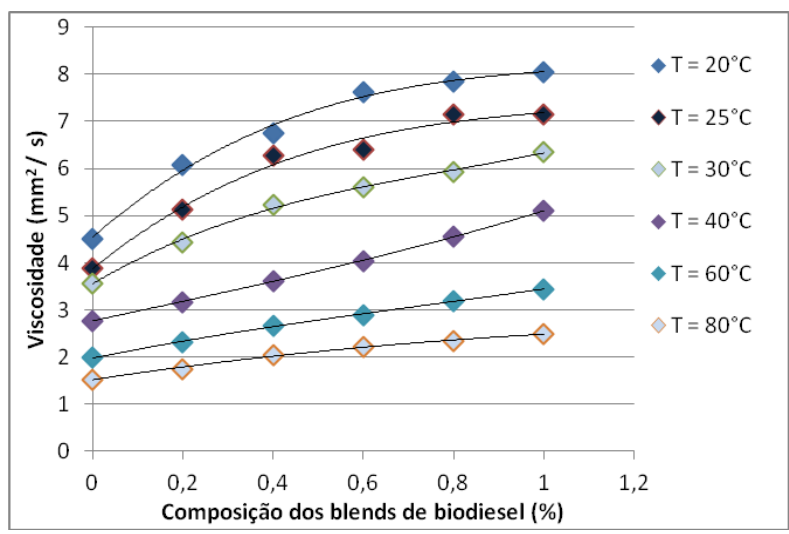

Figura 3 - Curva de viscosidade dos blends de diesel/ biodiesel de dendê. 


\subsection{Análise da Densidade}

A densidade do combustível é uma característica importante, pois de uma maneira geral, influencia a partida e pressão da injeção, além da pulverização do combustível, de modo a interferir no desempenho do motor de combustão e na emissão de gases, pois altas densidades podem gerar fumaça negra e emissão excessiva de material particulado. Os dados obtidos são apresentados nas tabelas 4, 5 e 6 :

Tabela 4: Densidade $\left(\mathrm{Kg} / \mathrm{m}^{3}\right)$ obtida para os blends biodiesel/diesel de Ouricuri.

\begin{tabular}{ccccccc} 
& B0 & B20 & B40 & B60 & B80 & B100 \\
\hline $\mathbf{1 5}{ }^{\circ} \mathbf{C}$ & 0,8453 & 0,8503 & 0,8583 & 0,8673 & 0,87175 & 0,8807 \\
$\mathbf{2 0}^{\circ} \mathbf{C}$ & 0,8405 & 0,8464 & 0,85375 & 0,8632 & 0,8679 & 0,87555 \\
$\mathbf{2 5}^{\circ} \mathbf{C}$ & 0,8388 & 0,8477 & 0,854 & 0,8588 & 0,8651 & 0,8705 \\
$\mathbf{3 0}^{\circ} \mathbf{C}$ & 0,8343 & 0,8432 & 0,8505 & 0,8557 & 0,8615 & 0,8665 \\
$\mathbf{4 0}^{\circ} \mathbf{C}$ & 0,8269 & 0,8359 & 0,8427 & 0,8483 & 0,8541 & 0,8587 \\
\hline
\end{tabular}

Tabela 5: Densidade $\left(\mathrm{Kg} / \mathrm{m}^{3}\right)$ obtida para os blends biodiesel/diesel de coco.

\begin{tabular}{rrrrrrr} 
& \multicolumn{1}{c}{$\mathrm{B} 0$} & \multicolumn{1}{c}{$\mathrm{B} 20$} & \multicolumn{1}{c}{$\mathrm{B} 40$} & \multicolumn{1}{c}{$\mathrm{B} 60$} & $\mathrm{~B} 80$ & \multicolumn{1}{c}{$\mathrm{B} 100$} \\
\hline $10^{\circ} \mathrm{C}$ & 0,8487 & 0,85715 & 0,85805 & 0,8655 & 0,8701 & 0,87315 \\
$15{ }^{\circ} \mathrm{C}$ & 0,8453 & 0,85345 & 0,8542 & 0,8621 & 0,8666 & 0,8693 \\
$20{ }^{\circ} \mathrm{C}$ & 0,8405 & 0,8491 & 0,8496 & 0,8574 & 0,862 & 0,8645 \\
$25{ }^{\circ} \mathrm{C}$ & 0,8388 & 0,8452 & 0,8463 & 0,8542 & 0,8575 & 0,8606 \\
$30{ }^{\circ} \mathrm{C}$ & 0,8343 & 0,8416 & 0,8437 & 0,8504 & 0,854 & 0,8574 \\
$40{ }^{\circ} \mathrm{C}$ & 0,8269 & 0,8371 & 0,83595 & 0,8442 & 0,84715 & 0,8502 \\
\hline
\end{tabular}

Tabela 6: Densidade $\left(\mathrm{Kg} / \mathrm{m}^{3}\right)$ obtida para os blends biodiesel/diesel de dênde.

\begin{tabular}{rccrccr} 
& B0 & B20 & \multicolumn{1}{c}{ B40 } & B60 & B80 & \multicolumn{1}{c}{ B100 } \\
\hline $\mathbf{1 5}^{\circ} \mathbf{C}$ & 0,8453 & 0,8547 & 0,862 & 0,8668 & 0,8704 & 0,873 \\
$\mathbf{2 0}^{\circ} \mathbf{C}$ & 0,8405 & 0,8505 & 0,858 & 0,8623 & 0,8662 & 0,8694 \\
$\mathbf{2 5}^{\circ} \mathbf{C}$ & 0,8388 & 0,8448 & 0,8512 & 0,8568 & 0,8619 & 0,866 \\
$\mathbf{3 0}^{\circ} \mathbf{C}$ & 0,8343 & 0,8412 & 0,8472 & 0,8532 & 0,8582 & 0,8622 \\
$\mathbf{4 0}^{\circ} \mathbf{C}$ & 0,8269 & 0,8337 & 0,8402 & 0,8458 & 0,8506 & 0,8546 \\
\hline
\end{tabular}

As Figuras 4, 5 e 6 apresentam os resultados obtidos a partir do estudo das viscosidades das misturas de diesel/ biodiesel das oleaginosas estudadas conforme as concentrações pré-estabelecidas: B20, B40, B60, B80, B100 e diesel 


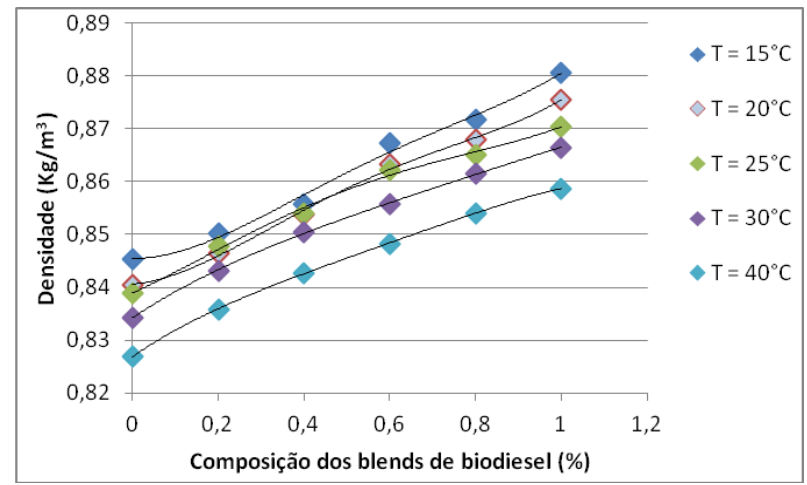

Figura 4 - Curva de densidade para as misturas de diesel/biodiesel de ouricuri.

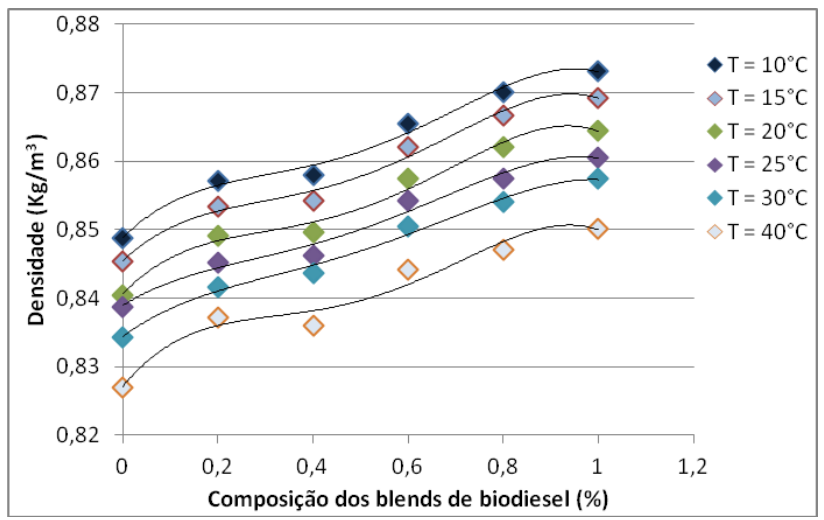

Figura 5 - Curva de densidade para as misturas de diesel/biodiesel de coco

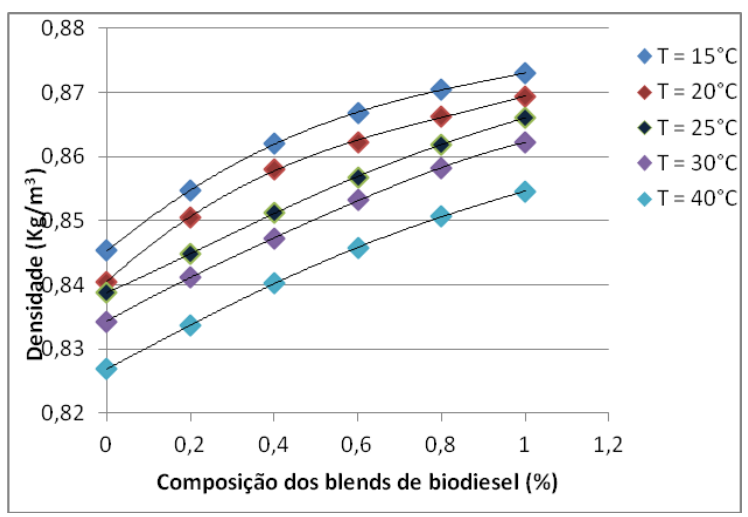

Figura 6 - Curva de densidade para as misturas de diesel/biodiesel de dendê

Analisando-se os resultados e comparando-os com a norma ASTM D664, cuja densidade deve estar entre $0,850-0,900 \mathrm{~kg} / \mathrm{m}^{3}$ à $20^{\circ} \mathrm{C}$, nota-se que o B100, que é o biodiesel puro, encontra-se dentro do valor exigido. A densidade do biodiesel de coco, ouricuri e dendê são ligeiramente mais elevadas 
que do diesel de petróleo. Portanto, aumentando o percentual de biodiesel nas mistura haverá um consequente aumento da densidade da mistura bidiesel/diesel. Em geral, a densidade de biodiesel é também afetada pelo aumento da temperatura: quanto maior a temperatura menor será sua densidade. A relação ar/combustível e o conteúdo de energia na câmara de combustão são influenciados pela densidade, o que afetaria o desempenho do motor.

\section{CONCLUSÃO}

A utilização das misturas de biodiesel e diesel vem sendo estudada detalhadamente, pois existem importantes propriedades que afetam o desempenho do motor podendo lhes causar algum tipo de dano. Neste trabalho, realizou-se uma investigação do efeito da temperatura nas analises de viscosidade e densidade para as misturas B0, B20. B40, B60, B80 e B100. Em geral, a viscosidade e a densidade de biodiesel são afetadas pelo aumento do percentual de biodiesel na mistura biodiesel/diesel e pelo aumento da temperatura: quanto maior a temperatura menor será sua viscosidade e densidade.

\section{REFERÊNCIAS}

AL-HAMAMRE, Z.; YAMIN,J. Parametric study of the alkali catalyzed transesterification of waste frying oil for Biodiesel production. Energy Conversion and Management 79 (2014) 246-254.

CAIRES, A.R.L.; LIMA, V.S.; OLIVEIRA, S.L. Quantification of biodiesel contente in diesel/biodiesel blends by fluorescence spectroscopy: Evaluantion of the dependence on biodiesel feedstock. Elsevier Renewable Energy 46 (2012) 137-140.

FERNANDES, D.D.S.; GOMES, A.A.; COSTA, G.B.; SILVA, G.W.B.; VERAS, G. Determinations of biodiesel contente in biodiesel/diesel blends using NIR and visible spectroscopy with variable selection. Elsevier Talanta 87 (2011) 30-34.

FREITAS, S. V. D.; SEGOVIA, J.J.; MARTÍN, M. C.; ZAMBRANO, J.; OLIVEIRA, M.B.; LIMA, A.S.; COUTINHO, J.A.P. Measurement and prediction of high-pressure viscosities of biodiesel fuels. Fuel 122 (2014) 223-228.

HOEKMAN, S.K.; BROCH, A.; ROBBINS, C.; CENICEROS, E.; NATARAJAN, M. Review of biodiesel composition, properties, and specifications. Elservier Renenwable and Sustainable Energy Reviews 16 (2012) 143-169..

SOUZA, J.E.; SCHERER, M. D.; CACERES, J.A.S.; CAIRES, A.R.L.; M’PEKO, J.C. A close dielectric spectroscopia analysis of diesel/biodiesel blends and potential dielectric approaches for biodiesel content assessment. Elsevier Fuel 105 (2013) 705-710. 